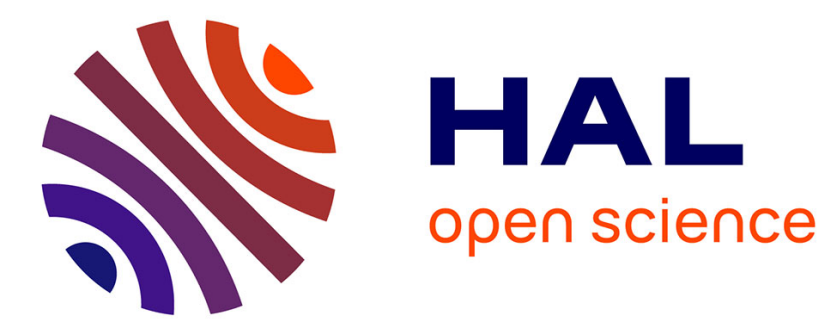

\title{
The Photomagnetoelectric Effect Dependencies on the Magnetic Field in the Ferromagentic CdCr2Se4
}

\author{
G. Sever, S. Yuseena
}

\section{To cite this version:}

G. Sever, S. Yuseena. The Photomagnetoelectric Effect Dependencies on the Magnetic Field in the Ferromagentic CdCr2Se4. Journal de Physique IV Proceedings, 1997, 07 (C1), pp.C1-277-C1-278. 10.1051/jp4:19971109 . jpa-00254748

\section{HAL Id: jpa-00254748 https://hal.science/jpa-00254748}

Submitted on 1 Jan 1997

HAL is a multi-disciplinary open access archive for the deposit and dissemination of scientific research documents, whether they are published or not. The documents may come from teaching and research institutions in France or abroad, or from public or private research centers.
L'archive ouverte pluridisciplinaire HAL, est destinée au dépôt et à la diffusion de documents scientifiques de niveau recherche, publiés ou non, émanant des établissements d'enseignement et de recherche français ou étrangers, des laboratoires publics ou privés. 


\title{
The Photomagnetoelectric Effect Dependencies on the Magnetic Field in the Ferromagentic $\mathrm{CdCr}_{2} \mathrm{Se}_{4}$
}

\author{
G.N. Sever and S.Yu. Yuseena
}

Department of Physics, Moscow Lomonosov State University, Moscow 119899, Russia

\begin{abstract}
The experimental results of the photomagnetoelectric effect (PMEE) dependencies on the magnetic field $H$ for film samples $\left(\mathrm{d} \sim 10^{-5} \mathrm{sm}\right)$ of chalcogenide spinel $\mathrm{CdCr}_{2} \mathrm{Se}_{4}$ (Curie point $\mathrm{T}_{\mathfrak{c}} \sim 129 \mathrm{~K}$ ) are presented in this work. The measurements of the PMEE have been conducted at different $\mathrm{H}$ (to $14 \mathrm{kOe}$ ) and temperature T (83-293 K). An analysis of the PMEE short-circuit current dependencies on $\mathrm{H}$ at different $\mathrm{T}$ and comparison with magnetic properties of the compound $\mathrm{CdCr}_{2} \mathrm{Se}_{4}$ show that in the ferromagnetic region the PMEE consists of the PMEE, which takes place at small $H$, and the PMEE, which is proportional to the external $H$. At Curie temperature the spontaneous effect disappears. In the far paramagnetic region exists only the ordinary PMEE. In the general case, the PMEE may be presented as sum of the ordinary PMEE, which is proportional to the magnetic induction $B$, and the anomalous PMEE, which is proportional to the magnetisation $M$, as in case of Hall-effect in ferromagnetics.
\end{abstract}

The photomagnetoelectric effect (PMEE) of Kikoin-Noskov is widely used for studying semiconductor materials and determining characteristics of the charge carriers. The receivers of radiation were constructed on its basis. However, the PMEE wasn't applied for investigation of magnetic semiconductors. Up to now the PMEE is observed only in [1].

In substances with magnetic ordering the PMEE can be different from one in ordinary semiconductors. In ferromagnetic the PMEE must be proportional to magnetic induction $B$, determining Lorenz force, but not the external magnetic field $H$. In general case the characteristics of the charge carriers, which influence on the PMEE magnitude (mobility, life time, surface recombination velocity), depend on the magnetic ordering in substances too.

Besides the ordinary PMEE, the anomalous PMEE (APMEE), which is connected with magnetic subsystem, can also exist, as in case of anomalous Hall effect in ferrites.

In this work there is a report about observation of the PMEE in ferromagnetic semiconductor films $\mathrm{CdCr}_{2} \mathrm{Se}_{4}$ (Curie point $T_{c} \sim 129 \mathrm{~K}$ ). There are results of the investigations of the PMEE dependencies on magnetic field $H$. On the basic of this date showed the existence of both the ordinary PMEE, which is proportional to induction $B$, and the PMEE, which is proportional to magnetisation $M$.

The measurements of the PMEE have been conducted on the films $\left(\mathrm{d} \sim 10^{-5} \mathrm{~cm}\right)$ with resistivity $\rho^{\sim 10^{3}} \Omega^{*} \mathrm{~cm}$ at room temperature. The geometry of experiment for the PMEE investigation was ordinary. The semiconductor films were located in magnetic field (field $\mathrm{H}$ was parallel to film plane) and were illuminated by strong-obsorbing light. Electromotive force of open circuit Voc or short-circuit current Isc PMEE were measured in direction, which was perpendicular to light beam and to magnetic field $\mathrm{H}$. The samples were illuminated by both monochromatic and nonmonochromatic light from the electric-filament lamp $(500 \mathrm{Wt})$. The frequency of light modulation was $80 \mathrm{~Hz}$. The measurements of the PMEE have been conducted at different $\mathrm{H}$ (to $14 \mathrm{kOe}$ ) and $\mathrm{T}(83 \div 293 \mathrm{~K}$ ). Contrary to monocrystal [1], in films the PMEE is found over a wide spectral range $(1.2 \div 3 \mathrm{eV})$ and approaches $1 \mathrm{~V}$.

The experimental data of the PMEE dependencies on magnetic field $H$ for film samples is shown on Fig. 1. The PMEE is odd effect for film and monocrystal. The PMEE dependencies on magnetic field $H$ in ferromagnetic $\mathrm{CdCr}_{2} \mathrm{Se}_{4}$ are essential nonlinear contrary to the ordinary PMEE.

For the film the current Isc is saturated at small H (Fig. 1 a). The character of dependence Isc( $H)$ in small $H$ can be associated with behaviour of the magnetisation $\mathrm{M}(\mathrm{H})$. The almost rectangular loop of magnetic hysteresis and small coercive force ( $\mathrm{Hc} \sim 4 \mathrm{Oe}$ ) are characteristic for the films. Besides, the demagnetising factor is negligibly small, if vector $H$ is parallel to film plane. The small magnetic field is enough for films to become monodomain. The spontaneous effect may be observed at these conditions. This effect in small H isn't observed in ordinary semiconductors.

The results of PMEE magnetic field dependencies at more strong magnetic field to $14 \mathrm{kOe}$ are shown on Fig. $1 \mathrm{~b}$. The PMEE current $I^{\text {sc }}$ changes linearly at increasing magnetic field $H$. So, the effect, which is proportional to field $H$, appears in this case. For film samples the experimental results show existence of two effects - the normal 
(ordinary) and anomalous PMEE. The normal PMEE is proportional field B, the APMEE is proportional to the magnetisation $M$.

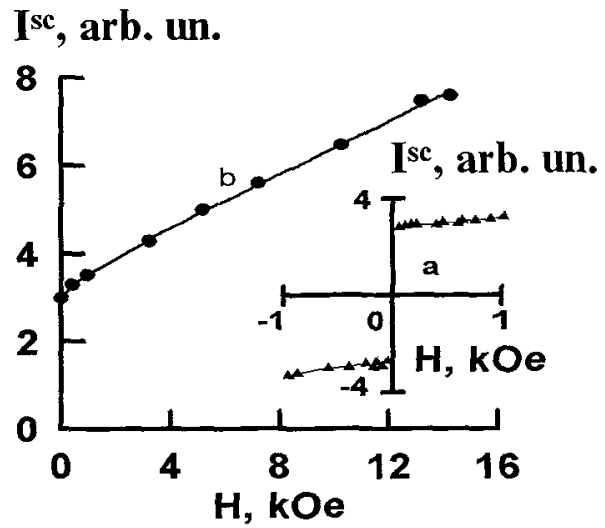

Fig. 1. Dependence of the short-circuit current PMEE Isc on the magnetic field $\mathrm{H}$ in film sample $\mathrm{CdCr}_{2} \mathrm{Se}_{4}$ a) for small $\mathrm{H}$ $<1 \mathrm{kOe}, \mathrm{b})$ for $\mathrm{H}<14 \mathrm{kOe}(\mathrm{T}=83 \mathrm{~K}, \mathrm{hv}=2,27 \mathrm{eV})$.

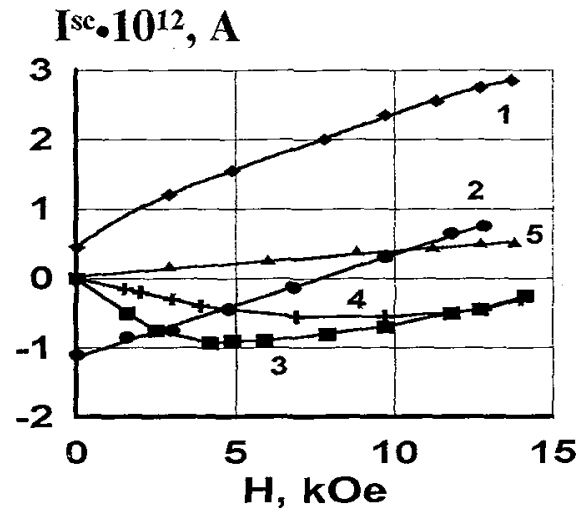

Fig. 2 Dependence of the short-circuit current PMEE Isc for film sample $\mathrm{CdCr}_{2} \mathrm{Se}_{4}$ (nonmonochromatic light) on the magnetic field $H$ for different temperature $T(1-83 \mathrm{~K}, 2$ $119 \mathrm{~K} ; 3-135 \mathrm{~K}, 4-143 \mathrm{~K}, 5-185 \mathrm{~K})$.

The results of PMEE investigations for different temperature $T$ are shown on Fig.2. The normal and anomalous PMEE exist in ferromagnetic region (83K-119K). The APMEE changes its sign with temperature rising and its absolute magnitude increases near $T \approx T c$. The dependencies $I^{s c}(H)$ is enough completely near $T \approx T_{c}$. The $P M E E$ is absent at $H=0$, but it appears in magnetic field. The magnetisation $M(H)$ is analogy dependence. The PMEE is proportional $\mathrm{H}$ in high temperature region. Thus, the ordinary PMEE is observed in far paramagnetic region (up to $293 \mathrm{~K}$ ). The temperature investigations are confirmed the connection PMEE with magnetic properties.

The reason of the APMEE is different from one of the Kikoin-Noskov effect [2]. The APMEE isn't connected with Lorenz force acting to moving charge carriers at magnetic field $B$. The explanation of the PMEE may be given on analogy with the explanation of the anomalous Hall-effect in ferromagnetics [3]. The effect is caused by scattering of the charge carriers on the fluctuations of spontaneous magnetisation. According to [3] in magnetic semiconductors the spontaneous effects can exist even without external magnetic field H. For its experimental observation it is necessary field $\mathrm{H}$ which is enough for making samples mononodomain. The reason of this effect may be interference of spin-orbit and exchange (or another) interactions. This interference leads to anisotropy of scattering probability of the charge carriers. It is necessary take into account presence of gradient concentration of charge carriers - holes and electrons in case of PMEE.

In general case PMEE in ferromagnetic can be present as sum of two parts:

$$
V=\alpha M+\beta B
$$

where $\alpha$ and $\beta$ - coefficients determining the magnitude of anomalous and ordinary effects accordingly. The analogy equation for the Hall-effect exists in ferromagnetics.

Our investigations of the PMEE in $\mathrm{CdCr}_{2} \mathrm{Se}$ show existence of APMEE in magnetic semiconductors. The ordinary PMEE was also discovered in film samples. The films of $\mathrm{CdCr}_{2} \mathrm{Se}_{4}$ are more interesting for the APMEE investigations and technical applications. The small coercive force and negligible small demagnetising factor (in case if $\mathrm{H}$ is parallel to film plane) allow to observe the PMEE at weak magnetic field. Besides, the films are more photosensitive in broad spectral region. The PMEE in magnetic semiconductors may be used for study of the influence of magnetic ordering on the kinetic and energy spectra of charge carriers.

\section{Acknowledgements}

The authors are grateful to V.P. Kononov for film samples and E.L.Nagaev for useful discussions.

\section{References}

[1] Sever G.N. Fiz. Tekh. Poluprov. 8 (1983) 1505.

[2] Kikoin I.K., Noskow M.N. Phys. Zs. Sowjet. 5 (1934) 586.

[3] Nagaev E.L., Physics of the magnetic semiconductors. (Moscow, 1973). 